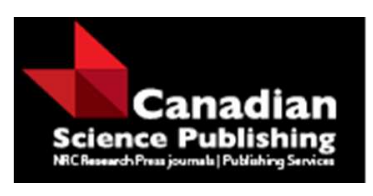

Canadian Journal of Physics

Revue canadienne de physique

\begin{abstract}
Second Born Fully Differential Cross Sections for Direct Ionization of $\mathrm{H}(1 \mathrm{~s})$ by Proton and Antiproton Collisions
\end{abstract}

\begin{tabular}{|r|l|}
\hline Journal: & Canadian Journal of Physics \\
\hline Manuscript ID & cjp-2015-0306.R3 \\
\hline Manuscript Type: & Article \\
\hline Date Submitted by the Author: & 04-Jul-2015 \\
\hline Complete List of Authors: & Tantawi, Reda; Zagazig University, Mathematics \\
\hline Keyword: & $\begin{array}{l}\text { Direct ionization, Closured second-order Born approximation, Fully } \\
\text { differential cross sections, Hydrogen atom, Proton and antiproton }\end{array}$ \\
\hline \multicolumn{2}{|l}{} \\
\hline
\end{tabular}

SCHOLARONE $^{\text {tu }}$
Manuscripts 


\title{
Second Born Fully Differential Cross Sections for Direct lonization of H(1s) by Proton and Antiproton Collisions
}

\author{
Reda S. Tantawi \\ Mathematics Department, Faculty of Science, Zagazig University, Zagazig, Egypt \\ E-mail: reda_tantawi@yahoo.com
}

\begin{abstract}
Fully differential cross sections (FDCS) for ionization of $\mathrm{H}(1 \mathrm{~s})$ atom by collision with proton and antiproton are calculated within the closured second-order Born approximation. The calculations are performed for emission of the electron with different energies into planes moving from the scattering to the perpendicular planes at different impact energies and small scattering angles where the significant contribution to the total cross section comes from. The influence of each of the projectile's charge and the second Born term on the calculations is varying according to the incident energy, emission energy, and the scattering angle.
\end{abstract}

Key words: Direct ionization, Closured second-order Born approximation, Fully differential cross sections, Hydrogen atom, Proton and antiproton.

PACS: $34.50 . F, 34.50 . P, 34.10 . X$ 


\section{Introduction}

One of the many diverse reactions which may take place as a consequence of collisions between a bare ion and an atomic target is that of single ionization, where an electron initially bound to the target is ejected into a continuum state with a well defined asymptotic value of the momentum. Reactions involving neutral atoms in the ground state play an important role in plasma, fusion and many other astrophysical processes. More precisely, an accurate knowledge of differential and total ionization cross sections for proton and antiproton collisions with atomic hydrogen has an important application in the diagnostics of fusion plasma [1,2]. Not only does this type of study provide valuable understanding of matter and anti-matter interactions, the antiproton opposite charge makes it the ideal particle for comparison to protons in searching for charge effects on fundamental theories of atomic physics [3]. Unlike electron impact collisions, the heavy-ion projectile is most probably scattered into a forward direction due to the small momentum transferred by the incident particle to the target electron.

In the case of heavy ion impact, it is important to separate ionization due to charge transfer to projectile bound states from those electrons directly ejected into the continuum, namely, the direct ionization reaction. However, for antiproton impact there is no ionization of the target due to the capture of target electron by the projectile.

The quantity of physical interest in the scattering process is the differential cross section [4]. The fully differential cross section measures the momentum distribution of the ionized electron for a fixed energy and scattering angle of the projectile.

In a simple physical picture, the Born series for the scattering amplitude can be regarded as a multiple scattering series in which the projectile interacts repeatedly with the interaction potential and propagates freely between two subsequent interactions. On the basis of this interpretation, the Born series can be expected to converge for non- 
relativistic scattering, if the incident particle is fast enough so that it cannot interact too many times with the potential and (or) if the interaction is sufficiently weak. The first detailed calculation on a total ionization cross section for proton-hydrogen system was carried out by Bates and Griffing [5] using the first-order Born approximation.

A simple formula for ionization cross sections was derived by Gryzinski [6]. Another formula, proposed by Gillespie [7, 8], gives results close to Gryzinski's formula at large energies, and makes corrections to Gryzinski's formula at small energies. Anyway, Gillespie's formula is not frequently used in applications, because it requires a knowledge of fitting parameters not always known a priori.

Omidvar [9] derived a general formula for the first-order Born approximation cross sections for ionization of hydrogen atom being initially in an arbitrary bound state. He applied this formula to study the ionization of atomic hydrogen by electron impact. Briggs [10] reported that the peaks structures in ionization fully differential cross section, that are present in first-order Born approximation, are due to a single binary collision between the projectile and the target electron, recoil of slow electrons from the target nucleus, and using a Coulomb wavefunction for the ionized electron instead of a plane wave. Also he reported that higher-order scattering processes, like second-order Born approximation, can lead to new binary structures and/or to changes in the shapes of cusps or peaks occurring in the first-order Born approximation.

Sahoo et al. [11] applied the first-order Born approximation in the impact parameter formalism to calculate the total and double differential ionization cross sections for proton and antiproton impact on atomic hydrogen in both the ground and $2 \mathrm{~s}$ states. They employed a final continuum state wave function which incorporates distortion due to the Coloumb fields of both the projectile and the target nuclei. 
The second-order Born approximation, employing the closure relation, is used by Maier and Liehl [12] for calculating angular distributions of electrons emitted from ground-state hydrogen atoms in collisions with fast protons. Voitkiv et al [13] used the first- and closured second-order Born, Glauber and the Continuum Distorted WaveEikonal Initial State approximations to calculate the fully differential cross section of ground-state hydrogen atoms in high-velocity collisions with bare ions. Harris et al. [14] found that an improved treatment of the ejected electron-ion interactions made little difference to the calculations, and it was demonstrated that the agreement between experiment and theory grew steadily worse as one moved from the scattering to the perpendicular plane.

Nassar and Tantawi [15] applied a closured second-order Born approximation to calculate the fully differential cross sections for ionization of hydrogen atoms, initially in the $2 \mathrm{~s}$ excited state, by proton and antiproton impact. Calculations were performed for electron emission in both the scattering and perpendicular planes. It was found that the dependence of the cross sections on the projectile charge increases as the energy of the ejected electron increases, and it plays an important role in the perpendicular plane, greater than it does in the scattering plane case.

In the present work, fully differential cross sections for ionization of ground-state hydrogen atoms by proton and antiproton impacts are calculated within the closured second-order Born approximation. I consider emission of the electron into the scattering plane, which contains the incoming projectile momentum and the momentum transfer, moving to the perpendicular plane, which is perpendicular to the scattering plane and contains the incoming projectile momentum. The influence of the charge of the projectile on the cross section is analyzed. 


\section{Calculations and discussions}

Direct ionization of hydrogen atoms by incident charged particles can be studied in a plane-wave treatment. For a heavy ion with charge $Z_{p}$ impinging with momentum $\mathbf{k}_{i}$ upon a hydrogen atom initially in the state $\psi_{i}(\mathbf{r})$ and scattered with momentum $\mathbf{k}_{f}$, the target electron undergoes a transition into a continuum state $\psi_{\mathbf{k}_{e}}(\mathbf{r})$ with momentum $\mathbf{k}_{e}$. The fully differential cross section depending on the energy $\varepsilon=\frac{1}{2} k_{e}^{2}$ and ejection angle $d \Omega_{e}$ of the electron, and of the direction of the outgoing projectile $d \Omega_{f}$ is given by (in atomic units) [16] :

$$
\frac{d^{3} \sigma}{d \varepsilon d \Omega_{e} d \Omega_{f}}=\frac{\mu^{2}}{(2 \pi)^{5}} \frac{k_{e} k_{f}}{k_{i}}\left|T_{f i}\right|^{2} .
$$

with $k_{i}=\mu v$. Here $v$ is the velocity of the incident particle and $\mu$ is the reduced mass of the ion-atom system. The energy conservation requires $k_{i}^{2}-k_{f}^{2}=\mu\left(k_{e}^{2}+I\right)$, where $I$ is the ionization potential of the target in Rydbergs. In the second-order Born approximation, the transition amplitude for the transition from eigenstate $i$ to eigenstate $f$ is given as $T_{f i}=T^{B 1}+T^{B 2}$.

For direct ionization process, the initial state of the system is written as a product of a plane wave with momentum $\mathbf{k}_{i}$ for the incident particle relative to the target nucleus and the initial state of the hydrogen atom, i. e.,

$$
\Psi_{i}=(2 \pi)^{-3 / 2} \exp \left[i \mathbf{k}_{i} \cdot \mathbf{R}\right] \psi_{i}(\mathbf{r})
$$

The final state wavefunction is a product of a plane wave with momentum $\mathbf{k}_{f}$ for the scattered particle relative to the target nucleus and the final continuum state of the 
target atom, i. e.,

$$
\Psi_{f}=(2 \pi)^{-3 / 2} \exp \left[i \mathbf{k}_{f} \cdot \mathbf{R}\right] \psi_{\mathbf{k}_{e}}(\mathbf{r})
$$

Within the first-order Born approximation, the projectile interaction with the target nucleus does not contribute to inelastic transitions, and the momentum transfer to the target is fully due to the single interaction between the projectile and the electron of the target atom. According to this approximation, the transition amplitude $T^{B 1}$ may be reduced to

$$
T^{B 1}=-\frac{4 \pi Z_{p}}{q^{2}} F(\mathbf{q})
$$

Here the form factor $F(\mathbf{q})$ defined by the expression

$$
F(\mathbf{q})=\left\langle\psi_{\mathbf{k}_{e}}^{(-)}(\mathbf{r})\left|\mathrm{e}^{i \mathbf{q} \cdot \mathbf{r}}\right| \psi_{i}(\mathbf{r})\right\rangle
$$

where $\mathbf{q}=\mathbf{k}_{i}-\mathbf{k}_{f}$ is the momentum transferred by the projectile to the electron. Because of the large mass difference between electrons and protons (antiprotons), it is clear that the projectile is unlikely to transfer more than a small fraction of its energy to the ejected electron. The final continuum wavefunction $\psi_{\mathbf{k}_{e}}^{(-)}(\mathbf{r})$ is represented by a Coulomb wavefunction, whose ingoing scattered part is defined as [16] :

$$
\psi_{\mathbf{k}_{e}}^{(-)}(\mathbf{r})=N\left(k_{e}\right) \mathrm{e}^{i \mathbf{k}_{e} \cdot \mathbf{r}}{ }_{1} F_{1}\left[-i / k_{e}, 1,-i\left(k_{e} r+\mathbf{k}_{e} \cdot \mathbf{r}\right)\right]
$$

with $N\left(k_{e}\right)=\mathrm{e}^{-\pi / 2 k_{e}} \Gamma\left(1+i / k_{e}\right)$ and ${ }_{1} F_{1}$ the confluent hypergeometric function. An analytical formula for the form factor (3) corresponding to an arbitrary initial atomic state is given in Ref. [13]. 
In the second-order Born approximation, the interaction between the projectile and the target nucleus must be taken into account. The expression for the second-order transition amplitude $T^{B 2}$ is given by

$$
T^{B 2}=\frac{4 \mu Z_{p}^{2}}{\pi} \sum_{n} \lim _{\eta \rightarrow 0^{+}} \int \frac{\left\langle\psi_{\mathbf{k}_{e}}^{(-)}(\mathbf{r})\left|\mathrm{e}^{i \mathbf{q}_{f} \cdot \mathbf{r}}-1\right| \psi_{n}(\mathbf{r})\right\rangle\left\langle\psi_{n}(\mathbf{r})\left|\mathrm{e}^{i \mathbf{q}_{i} \cdot \mathbf{r}}-1\right| \psi_{i}(\mathbf{r})\right\rangle}{q_{i}^{2} q_{f}^{2}\left(k_{n}^{2}-k^{2}-i \eta\right)} d \mathbf{k}
$$

where $\mathbf{q}_{i}=\mathbf{k}_{i}-\mathbf{k}$ and $\mathbf{q}_{f}=\mathbf{k}-\mathbf{k}_{f}$. The integration runs over the intermediate momentum $\mathbf{k}$ of the projectile, and the sum is taken over all the intermediate target states $\psi_{n}$, including the continuum ones. In Eq. (5) $k_{n}$ represents the corresponding projectile momentum. The matrix elements in expression (5) represents two separated virtual collisions between the projectile and the target atom. In the first step the projectile excites the target atom from the state $\psi_{i}$ to an intermediate state $\psi_{n}$ (bound or continuum) and transfers a momentum $\mathbf{k}_{i}-\mathbf{k}$ to the electron of the target atom. Secondly, the target is ionized into the continuum state with momentum $\mathbf{k}_{e}$ and the momentum transferred to the electron is $\mathbf{k}-\mathbf{k}_{f}$.

A simplified version of the second Born approximation, in which $k_{n}$ is replaced by $\bar{k}$ and the closure relation $\sum_{n}\left|\psi_{n}\right\rangle\left\langle\psi_{n}\right|=1$ is employed to perform the summation over the intermediate states, reduces the second order amplitude (5) to

$$
T^{B 2}=\frac{4 \mu Z_{p}^{2}}{\pi} \lim _{\eta \rightarrow 0^{+}} \int \frac{F(\mathbf{q})-\left[F\left(\mathbf{q}_{i}\right)+F\left(\mathbf{q}_{f}\right)\right]}{q_{i}^{2} q_{f}^{2}\left(\bar{k}^{2}-k^{2}-i \eta\right)} d \mathbf{k}
$$

According to Voitkiv et al. [13], if the scattering amplitude is evaluated in the frame work of second-order Born approximation, the cross section could only be calculated consistently to third order in the interaction potential, that is, 


$$
\left|T_{f i}\right|^{2} \approx\left|T^{B 1}\right|^{2}\left(1+2 \operatorname{Re} \frac{T^{B 2}}{T^{B 1}}\right) .
$$

which includes terms proportional to $Z_{P}^{2}$ or $Z_{P}^{3}$ only.

Since expression (6) contains two three-dimensional singularities at $\mathbf{k}=\mathbf{k}_{i}$ and $\mathbf{k}=\mathbf{k}_{f}$, and one dimensional singularity at $k=\bar{k}$, the corresponding integral is divided into two parts. One of them, which includes $F(\mathbf{q})$, can be calculated analytically using the results of Lewis [17]. The other part can be treated according to a semi-analytical method [18]. The singularity at $k=\bar{k}$ in the integrals can be transferred to another integral of a simple form using a subtraction procedure like that used in the method of Marchalant et al. [19].

In the present work, fully differential cross sections for direct ionization of ground-state hydrogen atoms by collision with protons and antiprotons at impact energies of 10, 20 and $100 \mathrm{keV}$ are calculated using formula (7). According to [13], the quantity $\bar{k}$ represents a free parameter. In the present work I found that $\bar{k}=k_{i} / 2$ yields the best fit, where the energy of the proton or antiproton projectile is $E=29.6 \times 10^{-6} k_{i}^{2} \mathrm{keV}$. The calculations are performed for several scattering angles, impact energies and ejected electron energies.

In the following figures, the fully differential cross sections correspond to emission of the electron in different planes are plotted against the emission angle $\theta_{e}$. Solid (dot) lines represent the results for emission into the scattering $\varphi_{e}=0^{o}$ (perpendicular $\varphi_{e}=90^{\circ}$ ) plane, and dash (dash dot) lines for emission into $\varphi_{e}=30^{\circ}$ ( $\varphi_{e}=60^{\circ}$ ) planes. Unfortunately, to the best of our knowledge, there is no 
corresponding experimental and other theoretical results has been reported to compare with.

Figure 1 illustrates the fully differential ionization cross sections of ground-state hydrogen atom, by proton (left panels) and antiproton (right panels) impact. All the plots correspond to a scattering angle of $0.5^{\circ}$ and an electron energy of $5 \mathrm{eV}$. From the comparison between the corresponding curves, it can be derived that the cross sections due to antiproton-induced reactions are much greater than those due to proton ones, being the differences between them smaller as the impact energy increases.

At $10 \mathrm{keV}$ impact energy (frames A and B), the behavior of the fully differential cross section for both proton (left panel) and antiproton (right panel) projectiles is quite different especially in the binary beak region for all the considered ejection planes. For proton scattering, the curves show maximum peaks with different heights in both the binary $\left(0^{\circ} \leq \theta_{e} \leq 180^{\circ}\right)$ and recoil $\left(180^{\circ} \leq \theta_{e} \leq 360^{\circ}\right)$ regions, being peaks located around $\theta_{e}=90^{\circ}$ much smaller than those around $\theta_{\mathrm{e}}=270^{\circ}$. Also, it can be seen that the fully differential cross sections decrease when moving from the scattering plane towards the perpendicular one, where the cross section seems to be symmetric around $\theta_{\mathrm{e}}=180^{\circ}$ and where the maximum peak of each curve is replaced by two minimum peaks. On the other hand, from the antiproton impact results, a minimum appears in the binary beak region whereas in the recoil angles the curves show their maximum values. In the binary region, it is seen that the cross sections, in contrast to proton, increased with moving from the scattering plane towards the perpendicular one. An opposite situation takes place for ejection into the recoil region which leads to flat and symmetric results in the perpendicular plane around $\theta_{\mathrm{e}}=180^{\circ}$. 
At $20 \mathrm{keV}$ (frames $\mathrm{C}$ and D), and $100 \mathrm{keV}$ (frames $\mathrm{E}$ and F) impact energies, the fully differential cross sections for both proton and antiproton projectiles show a very similar shape. The results exhibit minimum values in the binary region around $\theta_{e}=90^{\circ}$ and maximum peaks in the recoil region around $\theta_{e}=270^{\circ}$. It is clear that, the ejection into the scattering plane leads to large peaks, while moving towards the perpendicular plane reduces them. The cross sections for emission into the perpendicular plane are almost flat and symmetric about $\theta_{\mathrm{e}}=180^{\circ}$. Anyway, ejection of the electron into different planes will not influence the position of the peaks.

Fully differential cross sections for ionization of $\mathrm{H}(1 \mathrm{~s})$ by proton (left panels) and antiproton (right panels) impact, for an emission energy of $25 \mathrm{eV}$ and a scattering angle of $0.5^{\circ}$ are presented in figure 2. It is found that the contribution of the second-order transition amplitude, $T^{B 2}$, to the cross sections is similar to it on the results at $5 \mathrm{eV}$ ejection energy (figure 1) but with weaker effect. It can be noticed that the proton and antiproton impact cross sections, for ejection into the same plane, appear to describe approximately the same behavior. However, the effect of the projectile charge in the calculations at $25 \mathrm{eV}$ ejected electron energy (figure 2) is weaker than the one observed for calculations at $5 \mathrm{eV}$ (figure 1). The curves in the above figures show that the magnitude of heights of the recoil maximum peaks are greater than that of depths of the binary minimum peaks, especially for $0.5^{0}$ scattering angle. The cross sections for emission into the perpendicular plane are almost flat and symmetric about $\theta_{\mathrm{e}}=180^{\circ}$.

Similar calculations are performed considering a scattering angle of $1^{o}$ being the resulting cross sections are plotted in figures 3 and 4 , for 5 and 25 eV ejected electron energy, respectively. The curves show that the structure of the cross sections due to 
the proton and antiproton collisions with $\mathrm{H}(1 \mathrm{~s})$ seems to be nearly the same. It can be seen that in the cross sections binary minimum peaks, with different magnitudes, appear at around $90^{\circ}$ and $270^{\circ}$ ejected angles respectively. The magnitude of the peaks seems to decrease when moving from the scattering plane to the perpendicular one where the cross sections seems to be symmetric around $\theta_{e}=180^{\circ}$ and appear to be almost flat. The results due to the proton-induced reactions (left panels) are still smaller than those due to the antiproton impact (right panels). However, FDCS for proton and antiproton impact at $\mathrm{E}=100 \mathrm{keV}$ are almost comparable in shape as well as in magnitude.

In general, from the analysis of the preceding figures it could be said that the antiproton scattering process produces higher cross sections and that the effect of the sign of the charge of the projectile on the FDCS at $1^{o}$ scattering angle is smaller than those calculated for a scattering angle of $0.5^{\circ}$. Also, it could be said that the FDCS's for $0.5^{\circ}$ scattering angle are greater than those resulted obtained for $1^{o}$ scattering angle, suggesting the fact that, for heavy-ion impact, the projectile is most probably scattered near the forward direction due to small momentum transferred to the target electron. A comparison with first-order Born calculations, I have made, reveals that the secondorder transition amplitude, $T^{B 2}$, increases the cross sections for both proton and antiproton projectiles at $1^{o}$ scattering angle, while an opposite situation applies at $0.5^{\circ}$ scattering angle for the 5 and $20 \mathrm{keV}$ proton impact energies.

However, this effect on the cross sections at $1^{o}$ scattering angle is much greater than it at $0.5^{\circ}$ one, and it decreases with increasing the impact energy. A comparison with similar calculations for $\mathrm{H}(2 \mathrm{~s})$ atoms [15], illustrates that the structure of the fully 
differential cross section for both cases is quite different and the effect of the sign of the projectile's charge in the case of $\mathrm{H}(1 \mathrm{~s})$ target atom is more important. The contribution of the second-order transition amplitude to the FDCS in the case of $\mathrm{H}(2 \mathrm{~s})$ target atom is much greater than that in the case of $\mathrm{H}(1 \mathrm{~s})$ one. In such collisions, as the binding energy increases the interaction between the projectile and the electron of the ion in the process of excitation becomes much weaker than the interaction between the electron and the ionic nucleus. Therefore, it can be regarded as a weak perturbation.

\section{Conclusion}

A closured second-order Born approximation was employed to theoretically investigate the direct ionization of ground-state hydrogen atoms by proton and antiproton impacts. Ionization of the target electron into the scattering and the perpendicular planes as well as some other emission planes between them has been considered. The dependence of the calculations on the charge of the projectile increases with decreasing the scattering angle, and the contribution of the second Born term to the cross section in the antiproton projectile scattering process is greater than it in the proton one. The behavior of the results shows that the symmetric character of FDCS in the perpendicular plane is rigorous. Moving from the perpendicular plane to the scattering plane, generally, the symmetric character disappears. 


\section{References}

[1] C. D. Boley, R. K. Janev, and D. E. Post, Phys. Rev. A 52, 534 (1984).

[2]C. D. Boley, R. K. Janev, and D. E. Post, Nuc. Fus. 29, 359 (1989).

[3] H. Knudsen and J. F. Reading, Physics Reports 212, 107 (1992).

[4] J. S. Brigs and J. H. Macek, Adv. in At. Mol. and Opt. Phys. 28, 1 (1991).

[5] D. R. Bates and G. Griffing, Proc. Phys. Soc. London, Sect. A 66, 961 (1953).

[6] M. Gryzinski, Phys. Rev. A 138, 322 (1965).

[7] G. Gillespie, J. Phys. B: At. Mol. Phys. 15, L729 (1982).

[8]G. Gillespie, Phys. Lett. A 93, 327 (1983).

[9] K. Omidvar, Phys. Rev. 140, 26 (1964).

[10] J. S. Briggs, Comm. At. Mol. Phys. 23, 155 (1989) .

[11] S. Sahoo, K. Roy, N. C. Sil, and S. C. Mukherjee, Phys. Scr. 58, 126 (1998).

[12] C. H. Maier and H. J. Liehl, J. Phys. B: Atom. Molec. Phys. 10, 2841 (1977) .

[13] A. B. Voitkiv, B. Najjari and J. Ullrich J. Phys. B: At. Mol. Opt. Phys. 362591 (2003).

[14] A. L. Harris, D. H. Madison, J. L. Peacher, M. Foster, K. Bartschat, and H. P. Saha, Phys. Rev. A 75, 032718 (2007).

[15] T. E. I. Nassar and R. S. Tantawi Phys. Scr. 90, 015402 (2015).

[16] M. R. C. McDowell and J. P. Colemen, Introduction to the Theory of Ion-Atom Collisions (Amsterdam: North-Holand Publishing Company, 1970)

[17] R. R. Jr. Lewis, Phys. Rev. 102, 537 (1956). 
[18] M. Sahlaoui and M. Bouamoud, J. Phys. B: At. Mol. Opt. Phys. 45, 85201 (2012).

[19] J. P. Marchalant, C. T. Whelan and H. R. J. Walters, J. Phys. B: At. Mol. Opt. Phys. 31, 1141 (1998).

\section{Figures caption}

Figure 1: FDCS for proton (left panels) and antiproton (right panels) impact ionization of $\mathrm{H}(1 \mathrm{~s})$ at $0.5^{\circ}$ scattering angle and $5 \mathrm{eV}$ ejected electron energy, as a function of the polar emission angle $\theta_{e}$. The results for emission of the electron into the scattering plane (solid lines), $\varphi_{e}=30^{\circ}$ (dash lines), $\varphi_{e}=60^{\circ}$ (dash dot lines), and into the perpendicular plane (dot lines).

Figure 2. Same as Fig. 1, but for $25 \mathrm{eV}$ ejected electron energy.

Figure 3. Same as Fig. 1, but at $1^{o}$ scattering angle.

Figure 4. Same as Fig. 2, but at $1^{\circ}$ scattering angle. 

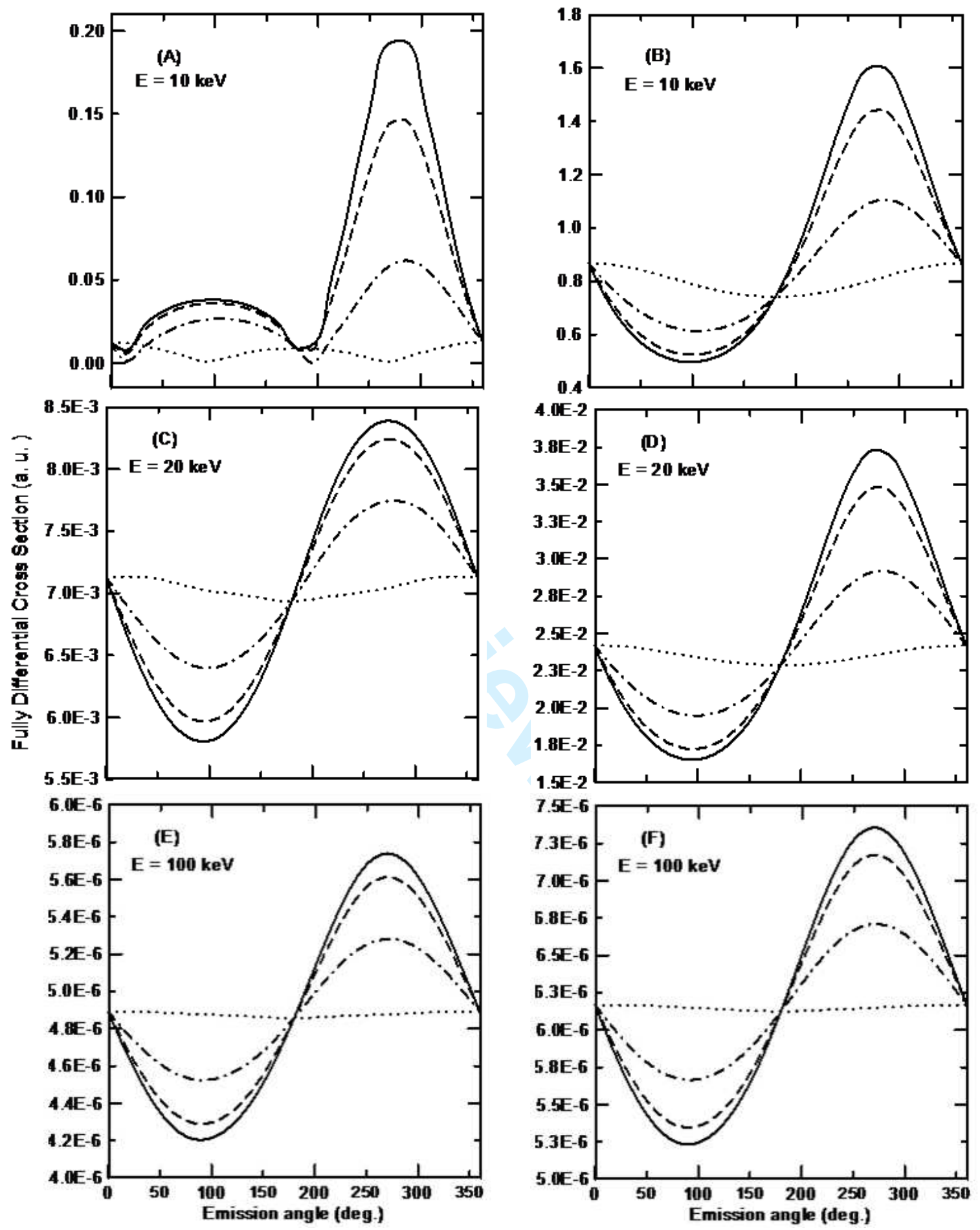

Fig. 1 

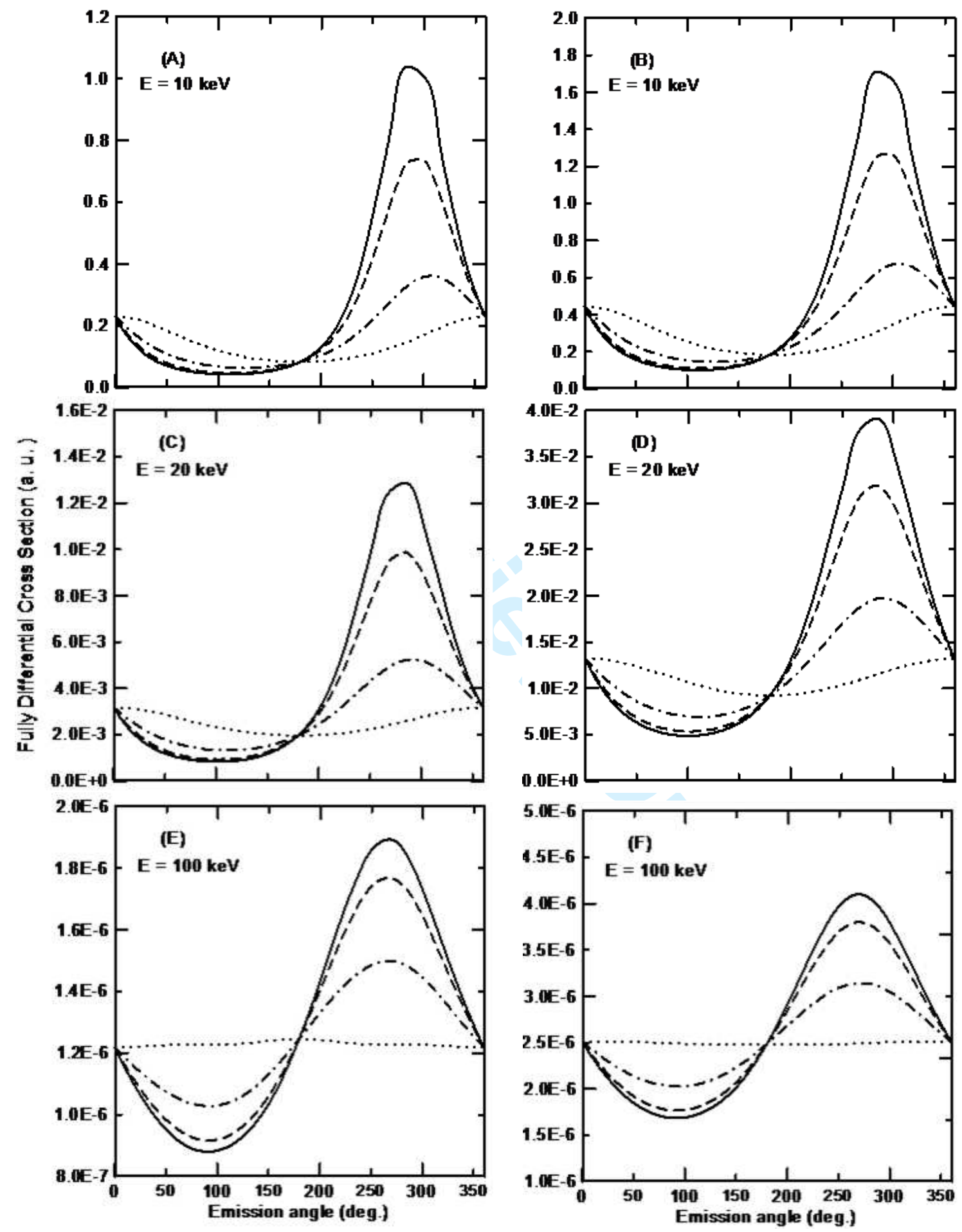

Fig. 2 

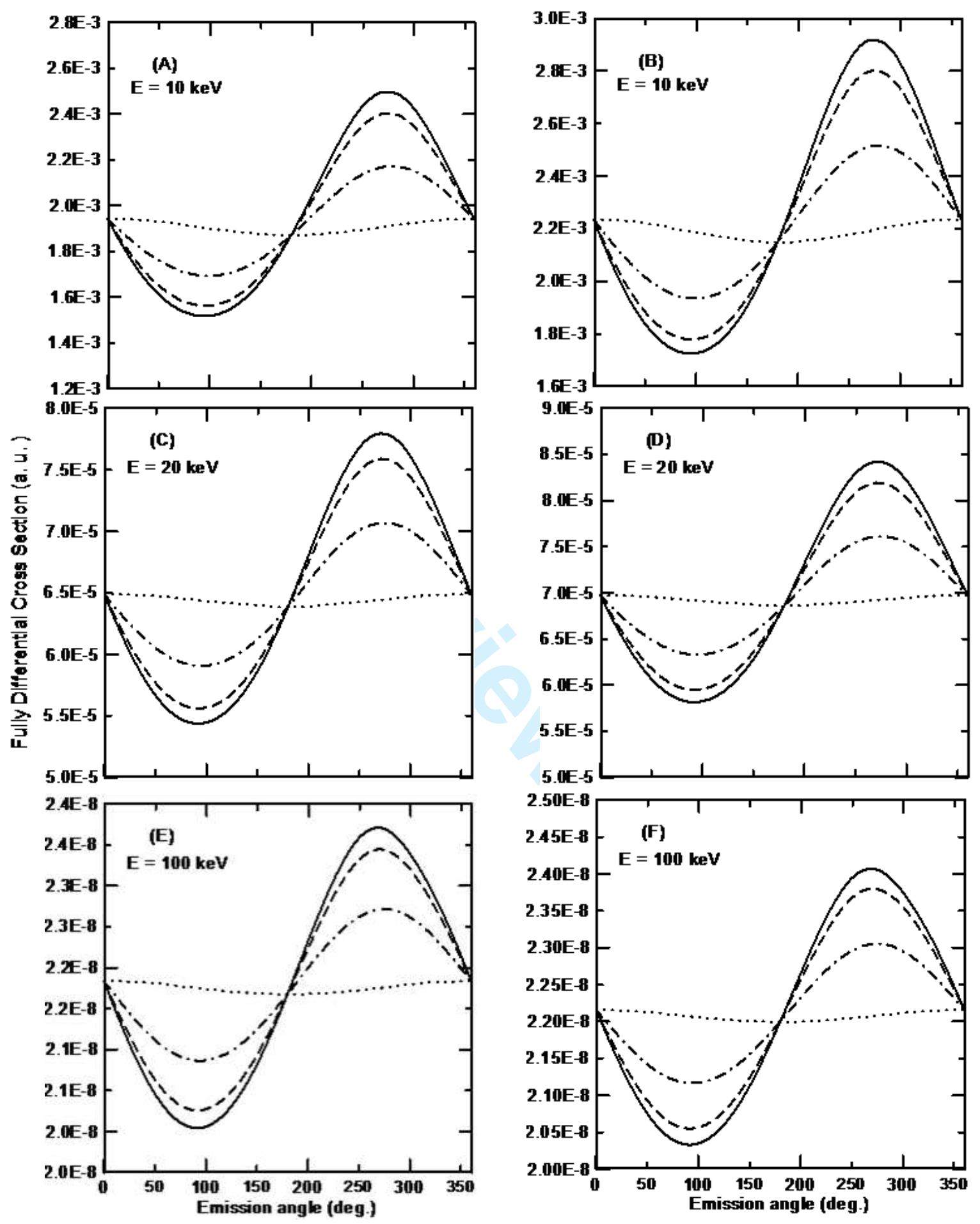

Fig. 3 

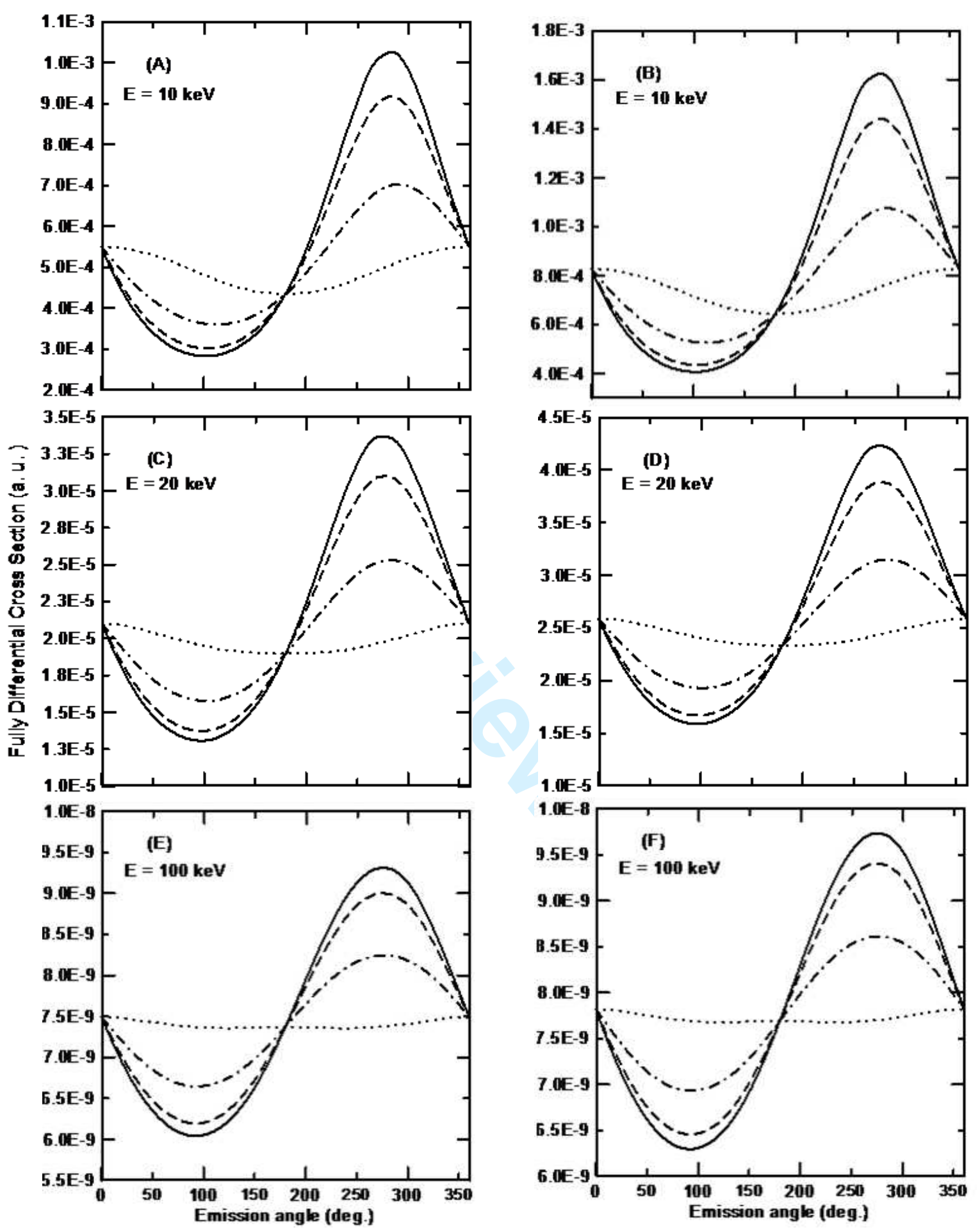

Fig. 4 